\title{
Fuel Consumption System
}

\author{
Yen-Jen Chen, Chia-Hung Chien \\ Ming Chi University of Technology, 84 Gungjuan Rd., Taishan Dist., New Taipei City 24301, Taiwan \\ Email: yjchen@mail.mcut.edu.tw, u97157091@gmail.com
}

Received April 2015

\begin{abstract}
Fleet Management System (FMS) is a highly applicable system which gets more and more attention among industrial field. Recent years, fuel consumption has become one of the most concerned topics, hence, establishment of a fuel consumption monitoring system is required. In this study, based-on the FMS, a system which was capable of precisely monitoring and calculating the fuel consumption was designed. This FMS was composed of the front end Vehicle Tracking System (VTS) and the back end Management Server (MS). VTS was established and installed into the vehicles, based on several well-known technologies, such as Mobile Telecommunications Technology of GPRS or 3G, Global Positioning System (GPS), and On-Board Diagnostics II (OBD-II). In addition, VTS was also connected with the Vehicle Electronic Control Unit (VECU) through the OBD-II interface. By using GPRS/3G technology and the TCP/IP communication protocol, real-time vehicle operation data obtained from the OBD-II and positioning information from the GPS could be sent backward to the MS. The MS was designed through JBoss Developer Studio, and included the Data Collector (DC) and Web server. The DC gathers data sent back from VTS and the Web server calculates and decides the vehicle's present situation according to the data. The result can also be presented on websites by the Web server. In this study, the fuel consumption status of vehicles could be performed. Furthermore, the Web service also provided the users instant online manipulation.
\end{abstract}

\section{Keywords}

Fleet Management System, Vehicle Tracking System, Fuel Consumption, Mobile Telecommunications, GPS, OBD-II

\section{Introduction}

The application of Fleet Management System (FMS) [1] is mainly attended to monitor multiple vehicles simultaneously. Although most of FMSs apply GPS [2] to monitor vehicles, there is no currently available system can provide real-time fuel consumption information and calculation service. For the aim of real-time monitoring and dynamic mobile data-recording, this study developed a fuel consumption calculating system, which combined Vehicle Tracking System (VTS), GPRS/3G, GPS and Web Server by using Machine to Machine (M2M) framework. In this design, the VTS could extract the necessary information from the vehicles, and with GPRS/3G and GPS techniques, it could achieve both data instantaneity and location preciseness. In addition, according to the Data Collector (DC) and Web of FMS Management Server (MS), this system could provide simple, convenient and fast service for the fleet managers to check the instant data online. Recent years, the International Crude Oil 
Price is often fluctuated, which results in the high gasoline price. Therefore, a system that could provide fuel consumption calculation and analysis is highly essential for the fleet managers.

On-Board Diagnostics (OBD) technique was first developed in 1975, called OBD-I, which is used for extracting data of fuel consumption and air pollution of vehicles; however, it is not widely used not only due to its connection interface set in the engine bay but also the insufficiency of error codes. In 1996, the more functioned version, OBD-II [3] was developed, which put its connection panel beneath the steering wheel and added many error codes, hence the OBD-II diagnosis panel was requested for all newly manufactured vehicles by the US government. In 2008, a mandate declared by government of R.O.C. indicated that all commercial vehicles should be equipped with OBD-II panel. Therefore, in Taiwan, cars manufactured after the year 2008 could obtain real-time fuel consumption data of the vehicles through the OBD-II and thus the acquirement of data were simplified and standardized.

Recent years, people increasingly rely on intelligent living style due to the maturation of GPS technique and the rapid development and improvement of communication technology. The technologies also improve the efficiency of enterprise management, in which the FMS is one of the benefited systems. Before the GPRS/3G and GPS technique applied in FMS, the fleet managers could only record, calculate and analysis car-driving data by paper-works. But after these two techniques applied in FMS, they could use VTS to read the VECU and extracted the detailed and precise information of a vehicle.

Meanwhile, the data could also be sent back to MS immediately and provide the fleet manager the first-hand report. This method not only increases the efficiency and preciseness of mobile data, but also decreases the prime cost of management through the M2M manner.

In this study, the calculation, analysis and data proof of fuel consumption were the most important parts and advantages. In Section 2, it will explain some methods about vehicle consumption calculation. In Section 3, it will introduce how Fuel Consumption System (FCS) integrates the VTS and MS and how VTS structure comprises the GPRS/3G, GPS and OBD-II system. The Section 4 will describe how to calculate and proof the fuel consumption right. The Section 5 will present the results of calculation and analysis and there is a summary of this study in Section 6.

\section{Related Works}

Recent years, due to the highly raised fuel price, more and more study tried to develop a system for calculation and analysis of fuel consumption. In the past, most FMSs conducting the calculation and analysis of fuel consumption was based on paper-records and mileage of the cars or the emission of carbonic molecules [4]. However, these two methods cannot provide instant and highly precise results. Calculating the fuel consumption through paper-records is a labor-intensive work and also hard to reach instantiation. Besides, this method might result in inaccuracy due to the lack of real-time data. Calculation of fuel consumption through detecting the carbon emission is more precise and useful, but the carbon emission may distinct from different types and ages of the engines; furthermore, application of different kinds of fuel can also result in distinctive carbon emission. Therefore, the types of engine, ages of engine and kinds of fuel can not only influence the amount of carbon emission but also affect the calculation results of fuel consumption.

OBD-II is an advanced technique applied for vehicle diagnosis. Most of recent studies used OBD-II to connect VECU and obtained the error code [5]. According to the error code, the managers could know the point of breakdown and could repair it. In addition, the OBD-II panel could also provide other detail information of the vehicle simultaneously, such as fuel status, coolant temperature, timing advance, absolute throttle position, etc. Based on the information, it could provide more precise and real-time analysis of fuel consumption.

\section{Architecture}

\subsection{Overall Architecture}

Overall architecture and application in this study is shown in Figure 1, and through the combination of VTS and MS, it provided remote monitoring and online calculation of fuel consumption. The following components are included to construct this system:

- VTS: is response to vehicle telematics monitoring and return vehicle information;

- Data Collector(DC): is for receiving and storing data;

- Web: is for calculating and analysing fuel consumption. 


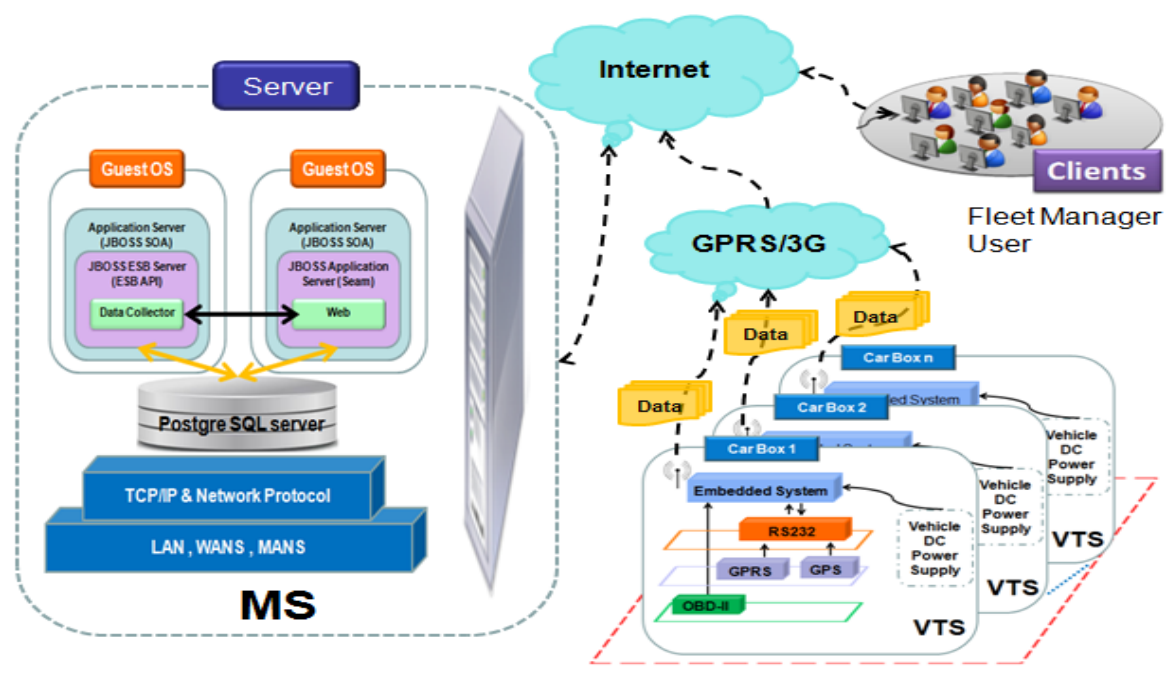

Figure 1. Overall architecture (VTS, MS).

The VTS sends the data backward to the DC of MS by the conduction of GPRS/3G, and the data format is transformed before being saved in the Database (DB), where Postgre SQL server is used to construct DB. Users could link to the Web Server through Internet and demand for the current fuel consumption information of their vehicles. The server would retrieve the data from DB for the demand of users and provide calculation and data analysis service. The results would all present on the website. The whole of MS was designed through JBoss Developer Studio [6].

\subsection{VTS Architecture}

VTS is used to achieve Vehicle telematics monitoring and vehicle information data return. The system components of VTS are shown in Figure 2.

- Embedded System (ES): is implemented by MCU (Micro Controller Unit) and responsible to the role of controlling and coordinating of other components;

- GPS receiver: is to receive GPS data and compute latitude and longitude position and time;

- OBD-II interface: is for the access to vehicle information (e.g., fuel status);

- GPRS/3G module: is to send vehicle information to server;

- SD card: is to store vehicle information.

ES connects to a vehicle by OBD-II interface, for obtaining fuel status. ES is integrated with GPS receiver and GPRS/3G module by RS232 interface and with SD card by SPI. Through GPS receiver, ES can obtain latitude and longitude position and GPS time. All vehicle information data is stored by SD card controlled by ES, and is sent to server through GPRS/3G module.

\section{Algorithm}

\subsection{Fuel Consumption Calculation Algorithm}

OBD-II is a national standard system for diagnosis of vehicles' fuel consumption and exhausts. It defines several numerical values, including coolant temperature, timing advance, absolute throttle position, fuel consumption, etc. Since January 2008, newly manufactured vehicles in Taiwan must be equipped with OBD-II panel, and this mandate has been performed much earlier in US. The OBD-II system is an event data recorder and connected to VECU to obtain internal data.

Fuel status in accordance with the OBD-II reads the fuel tank level height from VECU, each of scale behalf of the maximum capacity of the fuel tank $1 \%$. For a vehicle, to calculate fuel consumption statistics within a specified time range shall review the status of all the fuel returned within the time range (Figure 3). Before viewing and calculate fuel consumption we need to set two variables: "previous fuel value" and "initial fuel value". Initial fuel value is its first fuel value in the time range of fuel or the immediate fuel value after refuelling. Compared 


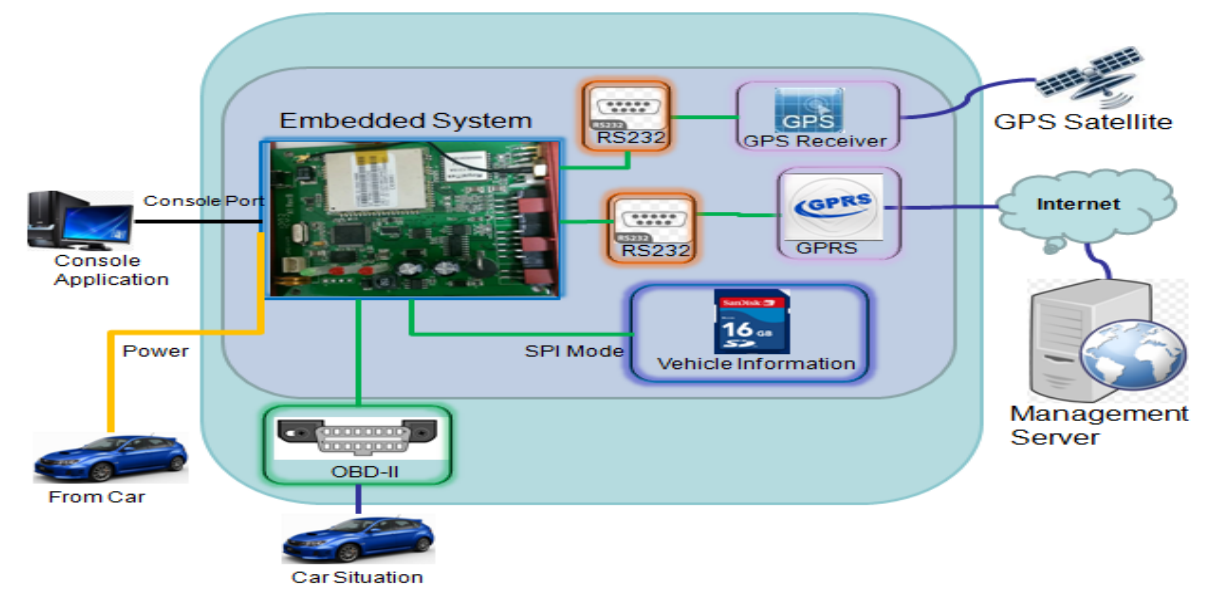

Figure 2. VTS architecture (GPRS, GPS, OBD-II, embedded system).

with the previous fuel value and the current fuel value, if the current fuel value is low, system will calculate the difference between current fuel and initial fuel values, and calculate fuel consumption. If the current fuel value is high, system will need to determine whether the fuel status is in refuelling or not. When the vehicle drives on the slope, the detected fuel amount may be higher or lower than the actual fuel amount, hence a variable, refuel detector, is applied for this circumstance. The refuel detector could be used for refuelling detection in order to rule out the false increase of the fuel. The threshold of refuel detector was set in 5 times, which indicates the fuel status is in refuelling when consecutive five times of increase of the fuel happens. According to the result in this study, five times was an acceptable and precise number for the refuel detector.

\subsection{Fuel Consumption Analysis Algorithm}

Single vehicle consumption analysis algorithm (Figure 4(a))

1) Set the query conditions: license number (single), time, fuel type, compute cycles

2) Get corresponding to the vehicle information from the database, and sort by time of data

3) According to the user specified time range, calculate and analyse the vehicle related to fuel consumption information (average speed, average Revolution(s) Per Minute (RPM), fuel consumption, efficiency of fuel consumption $(\mathrm{Km} / \mathrm{L})$, driving distance...)

4) Show the calculation and analysis of the results to chart

5) Multiple vehicle consumption analysis algorithm (Figure 4(b))

6) Set the query conditions: license number (plurality), time, fuel type, compute cycles

7) Get corresponding to the vehicle information from the database, and sort by time of data

8) In accordance with license number, transmits the acquired data to classify

9) Separate calculation and analysis of each vehicle related to fuel consumption information (average speed, average RPM, fuel consumption, efficiency of fuel consumption $(\mathrm{Km} / \mathrm{L})$, driving distance...)

10)Show the calculation and analysis of the results to chart

\section{Results of Fuel Consumption Analysis}

\subsection{Single Vehicle Consumption Analysis}

In Figure 5(a), according to the analysis of fuel consumption, especially for the different types of consumption including total fuel consumption, fuel consumption for mileage and fuel consumption for idle, the results can provide the information about the driver's driving habit. The analysis of fuel consumption for idle can reveal that if the driver performed improper engine idling which largely increased fuel consumption as well as the environmental pollution and fuel wasting.

\subsection{Multiple Vehicle Consumption Analysis}

In Figure 5(b), through the comparison between different drivers or vehicles, this system can tell apart the 


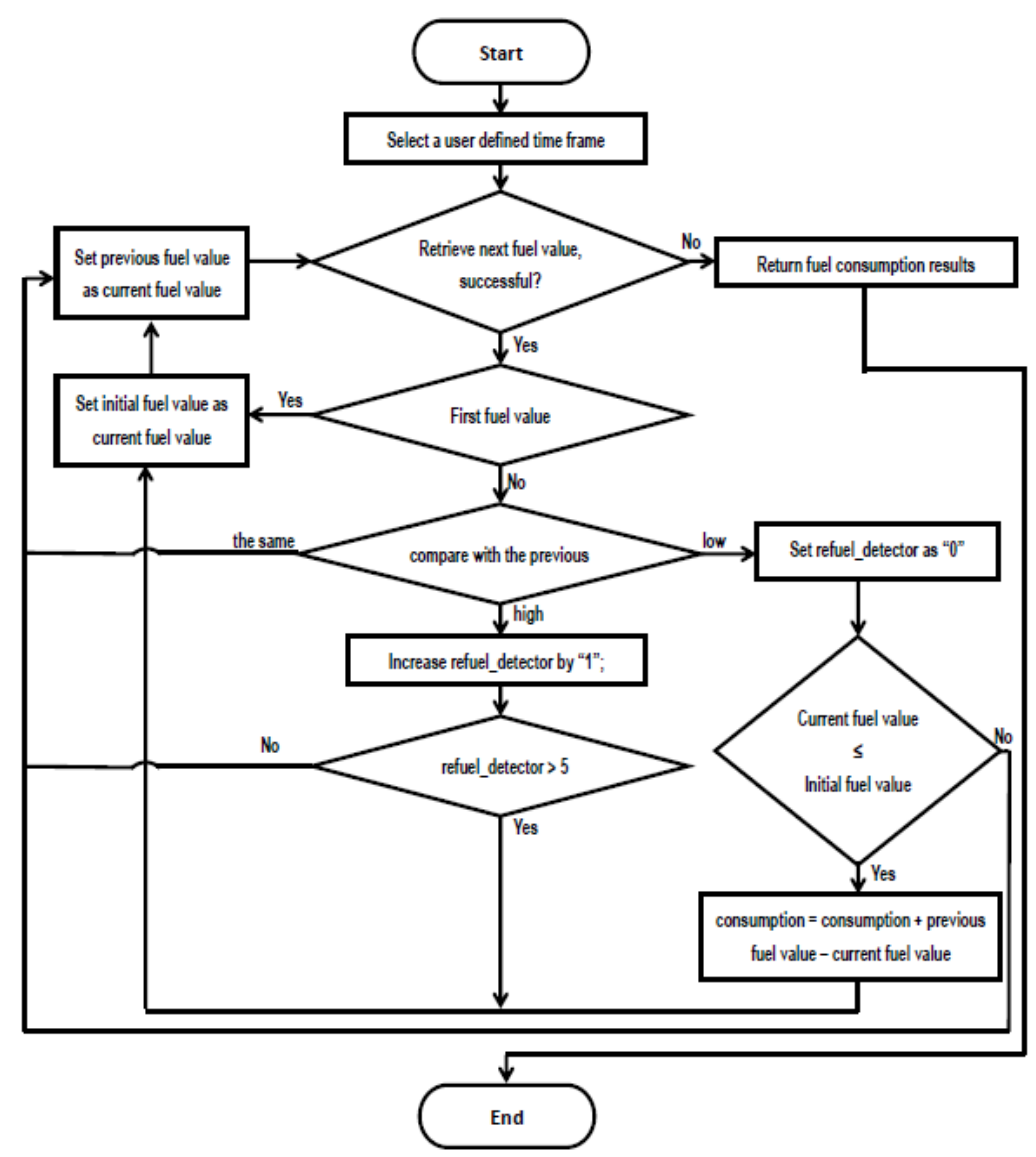

Figure 3. Fuel consumption calculation algorithm.

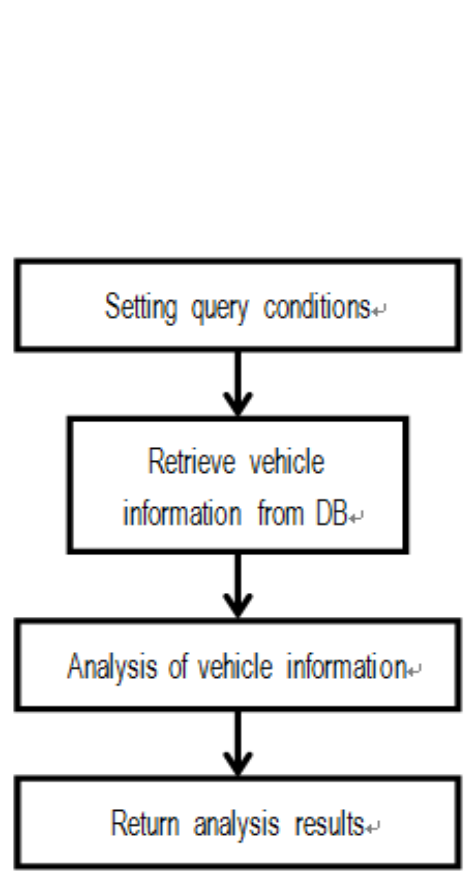

(a)

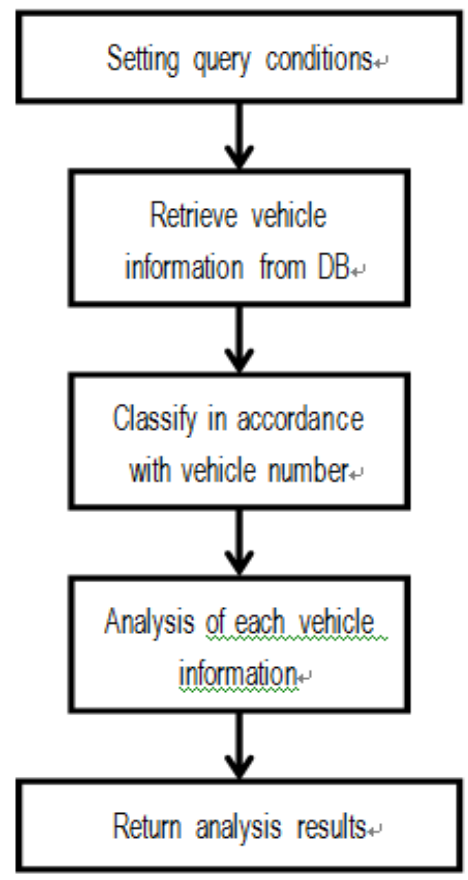

(b)

Figure 4. Fuel consumption analysis algorithm: (a) for a single vehicle; (b) for multiple vehicles. 


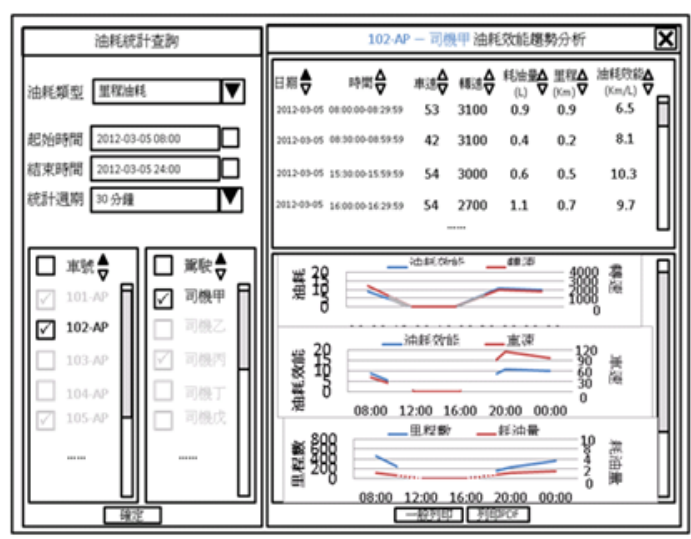

(a)

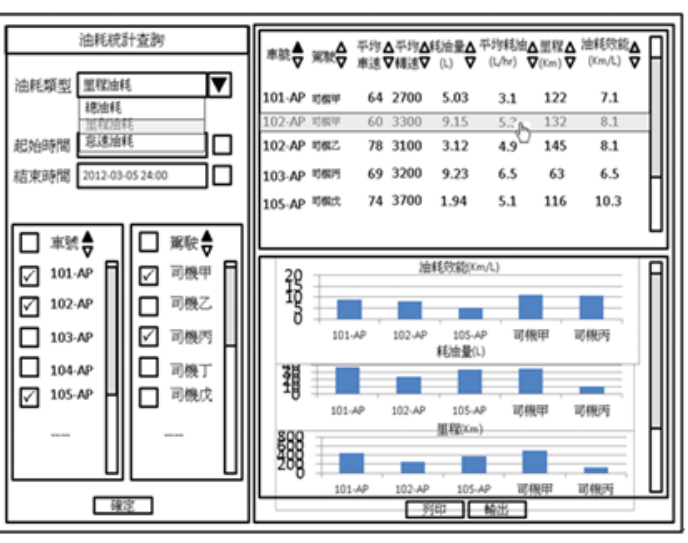

(b)

Figure 5. Results of fuel consumption analysis: (a) for a single vehicle; (b) for multiple vehicles.

problematic driver or vehicle by the analytic charts. If different drivers drive the same car and the results always show more fuel consumption for the car, the excessive consumption should come from the poor vehicle. Whereas, if the same driver drives different cars and the results always exhibit more fuel consumption for the driver, the excessive consumption is more likely coming from the inadequate driving habit of the driver.

\section{Summary}

Due to the rapid development of M2M technique, it is cost-saving and can also provide data instantaneity and preciseness to use M2M as the core structure. In this study, the designation comprises not only the M2M technique, but also the commonly applied GPRS/3G, GPS, and OBD-II, which improves the function of FMS and increases the efficiency of industrial management.

The GPRS/3G sends back the real-time OBD-II data to FMS Management Server (MS). The MS combined with the Fuel Consumption calculating system developed in this study can calculate and analyse the fuel consumption precisely in real time. By using this system, the fleet manager can provide proper adjustment to each vehicles and drivers according to the results analysis, and decrease the managing costs and increase the efficiency of management.

The further study will focus on extracting much more mobile data from this system and use the MS to analysis the mobile status, which will much improve the vehicle management and road safety.

\section{References}

[1] Gutiérrez, V., Izaguirre, M., Pérez, J., Munoz, L., López, D. and Sánchez, M. (2010) Ambient Intelligence in Intermodal Transport Services: A Practical Implementation in Road Logistics. The 4th International Conference on Sensor Technologies and Applications (SENSORCOMM), Venice, 18-25 July 2010, 203-209.

[2] Thong, S.T.S., Han, C.T. and Rahman, T.A. (2007) Intelligent Fleet Management System with Concurrent GPS \& GSM Real-Time Positioning Technology. The 7th International Conference on ITS Telecommunications, Sophia Antipolis, 6-8 June 2007, 1-6.

[3] OBD-II (On-Board Diagnostics-II) on http://www.obdii.com/

[4] Liu, J. and Wang, Y. (2011) Development on Automobile Fuel Consumption Intelligent and Rapid Testing System. International Conference on Electric Information and Control Engineering (ICEICE), Wuhan, 15-17 April 2011, 49424945.

[5] Jhou, J.-S., Chen, S.-H., Tsay, W.-D. and Lai, M.-C. (2013) The Implementation of OBD-II Vehicle Diagnosis System Integrated with Cloud Computation Technology. The 2nd International Conference on Robot, Vision and Signal Processing (RVSP), Kitakyushu, 10-12 December 2013, 9-12. http://dx.doi.org/10.1109/RVSP.2013.55

[6] JBOSS on http://www.jboss.org/ 